\title{
Rabdomiolisis por levetiracetam
}

\section{Rhabdomyolysis by levetiracetam}

\author{
Sebastián Felipe Sierra, Jessica Tatiana Olarte, \\ Dolli Yineth CÁceres • Bogotá, D.C. (Colombia)
}

\section{Resumen}

Introducción: el levetiracetam (LEV) es un medicamento anticonvulsivo con mecanismo de acción desconocido. La mayoría de efectos adversos son de intensidad leve a moderada, siendo la rabdomiolisis (RBM) uno recientemente descrito, con pocos casos reportados en el mundo.

Descripción del caso: hombre de 35 años, con antecedentes de obesidad y epilepsia generalizada sin tratamiento, quien cursa con estatus epiléptico, controlado con benzodiacepina y LEV satisfactoriamente. Examen neurológico y tomografía de cráneo simple descartan alteraciones asociadas. Presenta lesión renal aguda por RBM que a pesar de estatus epiléptico controlado y manejo con soporte dialítico, progresan hacia el deterioro. Se sospecha RBM por LEV luego de descartarse etiología autoinmune y RBM por inmovilización de paciente obeso. Se suspende el LEV observándose descenso rápido de la creatina quinasa (CQ) y mejoría progresiva de la lesión renal aguda, confirmándose así la sospecha diagnóstica. Se logra destete y retiro de soporte dialítico, dándose alta hospitalaria con función renal y CQ normales, ácido valproico como anticonvulsivante y alerta de contraindicación para manejo con LEV.

Discusión: el LEV es un antiepiléptico cuyos efectos adversos más frecuentes son: somnolencia, mareo, irritabilidad, cefalea y fatiga. La RBM por LEV es un efecto adverso infrecuente (pocos casos reportados en el mundo y ninguno reportado en Colombia). La sociedad europea incluyó dentro de los rótulos del LEV este efecto adverso recientemente. Su tratamiento se basa en suspender el medicamento y administrar terapia de soporte, así como, seguimiento clínico estrecho.

Conclusión: la RBM por LEV es un efecto adverso poco frecuente que debe sospecharse en presencia de lesión renal aguda por RBM sin otras causas explicables.

Palabras clave: levetiracetam, rabdomiolisis, lesión renal aguda, creatina quinasa.

\section{Abstract}

Introduction: levetiracetam (LEV) is an anticonvulsant medication with an unknown mechanism of action. The majority of adverse effects are of mild to moderate intensity, being rhabdomyolysis (RBM) one recently described, with few cases reported in the world.

Description: the case of a 35-year-old man with a history of obesity and generalized epilepsy without treatment is presented. He had an epileptic status controlled with benzodiazepine and LEV satisfactorily. Neurological examination and simple skull tomography ruled out associated alterations. He had acute renal injury due to RBM that despite controlled epileptic status and management with dialysis support, progressed towards deterioration. RBM by LEV was suspected after having discarded autoimmune etiology and RBM by immobilization of obese patient. The LEV was suspended showing a rapid decrease in creatine kinase (CQ) and progressive improvement of the acute renal lesion, thus confirming the diagnostic suspicion. Weaning and withdrawal of dialysis support was achieved, and was discharged from hospital with normal renal function and CQ, prescribing valproic acid as anticonvulsant and a contraindication warning for management with LEV.

Discussion: LEV is an antiepileptic whose most frequent adverse effects are: drowsiness, dizziness, irritability, headache and fatigue. The RBM by LEV is an infrequent adverse effect (few cases reported in the world and none reported in Colombia). European society included this adverse effect recently within the LEV labels. Its treatment is based on suspending the medication and administering support therapy, as well as close clinical follow-up.

Conclusion: RBM by LEV is a rare adverse effect that should be suspected in the presence of acute renal injury due to RBM without other explainable causes.

Key words: levetiracetam, rhabdomyolysis, acute kidney injury, creatine kinase
Dr. Sebastián Felipe Sierra Umaña: Residente de Medicina Interna, Departamento de Medicina Interna, Universidad Nacional de Colombia; Jessica Tatiana Olarte y Dolli Yineth Cáceres Galíndez: Estudiantes de Medicina, Universidad Nacional de Colombia, Facultad de Medicina. Bogotá, D. C. (Colombia). Correspondencia: Dr. Sebastian Felipe Sierra, Bogotá, D. C. (Colombia). E-mail: sfsierrau@unal.edu.co 


\section{Introducción}

El levetiracetam (LEV) es un anticonvulsivante utilizado en varios tipos de epilepsia. Su mecanismo de acción es desconocido. Sin embargo, el LEV se une a la proteína sináptica SV2A, la cual ha sido asociada en modelos animales con epilepsia $(1,2)$. Hay evidencia que sugiere que el LEV modula indirectamente receptores $\operatorname{GABA}(3,4)$ partial seizure reduction ( $>50 \%$. Es un medicamento relativamente bien tolerado. La mayoría de efectos adversos son de intensidad leve a moderada y ocurren más frecuentemente al inicio del tratamiento $(5,6)$. La rabdomiolisis $(\mathrm{RBM})$ por LEV es un efecto adverso recientemente descrito, con pocos casos reportados en el mundo (7-11). Aquí, se describe un caso de RBM por LEV en un paciente masculino de 35 años, $y$ se realiza una breve revisión de la literatura.

\section{Descripción del caso}

Hombre de 35 años, con antecedentes de obesidad grado III y epilepsia generalizada primaria desde los 13 años sin tratamiento, quien presenta pérdida súbita del estado de conciencia y movimientos tónicoclónicos de las cuatro extremidades de 20 minutos de duración. En valoración inicial se considera estatus epiléptico, realizándose intubación orotraqueal, infusión de benzodiacepina, impregnación con LEV y estudio tomográfico de cráneo simple, este último sin alteraciones. Es remitido a unidad de cuidados intensivos (UCI) bajo efectos de sedoanalgesia con RASS - 1, documentándose al examen físico: tensión arterial 169/86 $\mathrm{mmHg}$, frecuencia cardiaca $94 \mathrm{lpm}$, frecuencia respiratoria $18 \mathrm{rpm}$, saturación de oxígeno 97\% con fracción inspirada de oxígeno de 0.5 , temperatura axilar de $37.6^{\circ} \mathrm{C}$, murmullo vesicular disminuido simétrico bilateral, isocoria de $2 \mathrm{~mm}$ reactivas a la luz, simetría facial, lengua y úvula centrales, sin papiledema a la fundoscopia, movilizando simétricamente las cuatro extremidades, reflejos musculotendinosos
+/++++ simétricos, sin signos meníngeos ni cerebelosos. Paraclínicos evidencian elevación de azoados y creatina quinasa (CQ), considerándose lesión renal aguda secundaria a mioglobinuria por RBM en contexto de estatus epiléptico. Se indica manejo con lactato de ringer, ajuste de medicamentos a función renal y se alerta al no uso de nefrotóxicos durante seguimiento. Paciente continúa en deterioro de función renal y elevación de CQ, por lo que se inicia soporte dialítico y toma de electroencefalograma con sospecha de estatus epiléptico no controlado, el cual se descarta. Se considera como nueva sospecha diagnóstica RBM por inmovilización en paciente obeso, continuándose por tanto soporte dialítico, seguimiento clínico y paraclínico estrecho, al tiempo que se descarta etiología autoinmune y/o infecciosa. Sin embargo, por CQ persistemente elevada en presencia de soporte dialítico se decide realizar electromiografía de cuatro extremidades y suspender LEV por sospecha de RBM medicamentosa. Electromiografía describe enfermedad primaria de fibra muscular en fase aguda compatible con miopatía inflamatoria. Se observa descenso progresivo y rápido de la $\mathrm{CQ}$, así como mejoría de la función renal posterior al retiro del LEV (Figuras 1 y 2), confirmándose así, RBM por LEV.

Paciente continúa con evolución hacia la mejoría, en titulación de ácido valproico como nuevo anticonvulsivante, con destete exitoso del soporte dialítico y normalización de niveles azoados, por lo que es dado de alta para continuar manejo ambulatorio de epilepsia y obesidad. Se deja consignado en historia clínica contraindicación para consumo de LEV por efecto adverso mayor.

\section{Discusión}

El LEV es un antiepiléptico usado para el manejo de crisis convulsivas en adultos y niños. Dentro de sus efectos adversos más frecuentes se encuentran somnolencia, mareo, irritabilidad, cefalea y fatiga, los cuales no son dosis

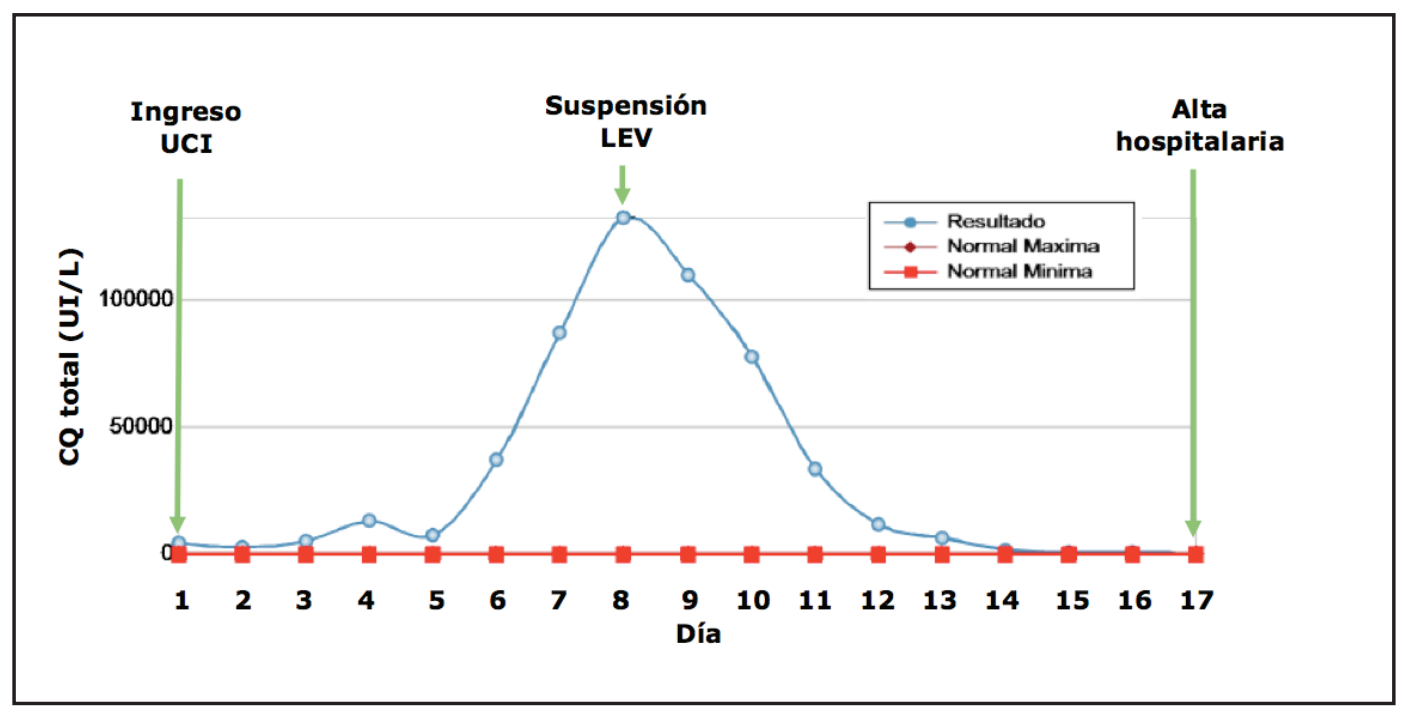

Figura 1. Evolución creatina quinasa (CQ). Eventos destacados (flechas verdes). Fuente: Documento obtenido durante realización del estudio. 


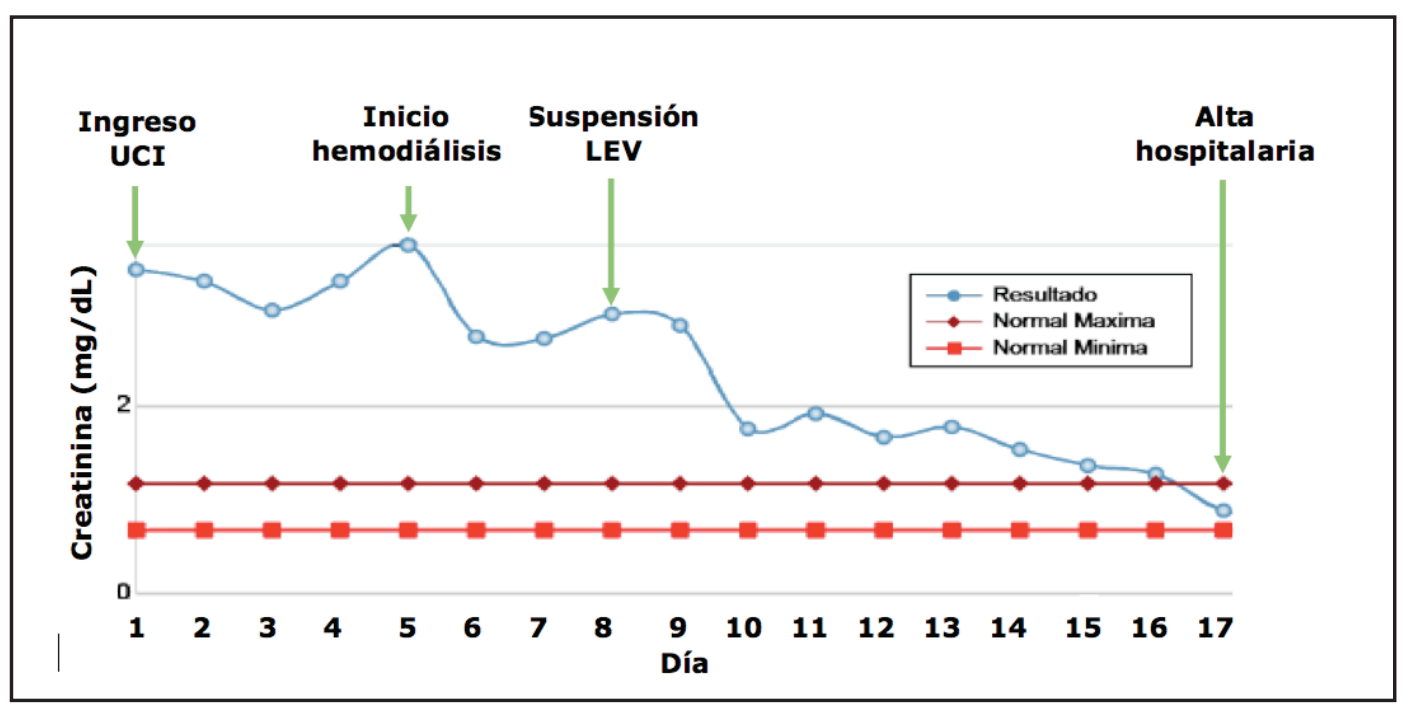

Figura 2. Evolución creatinina en suero. Eventos destacados (flechas verdes). Fuente: Documento obtenido durante realización del estudio.

dependiente (7). En Japón los efectos adversos reportados por LEV incluyen nasofaringitis $53 \%$, somnolencia $35.5 \%$, cefalea $19.9 \%$, mareos $17.5 \%$, diarrea $13.8 \%$, y estreñimiento $10.9 \%$ (7). La RBM como efecto adverso a este medicamento es infrecuente, con pocos casos reportados a nivel mundial (7-11). El mecanismo por el cual el LEV causa RBM se desconoce, sin embargo, se ha propuesto que este activa procesos de fusión y necrosis de células musculares esqueléticas $(7,12)$. La incidencia de RBM por antiepilépticos como grupo de medicamentos, es igualmente baja. Suele observándose más frecuentemente con el uso de otros medicamentos tales como: estatinas, fibratos, antibióticos (por ejemplo, daptomicina), neurolépticos y medicinas tradicionales chinas (13-16). Es por esto, que el caso aquí presentado toma gran importancia como reto diagnóstico y terapéutico, al ser la RBM por LEV una entidad que debe considerarse en pacientes con lesión renal aguda y elevación de CQ persistentes. En Colombia no se han reportado casos de RBM por LEV, siendo por tanto el caso aquí reportado el primer aporte a la literatura local.

La presencia de lesión renal aguda con CQ elevada en pacientes con estatus epiléptico, indica sospechar como primera posibilidad RBM por lesión mecánica muscular propia de los eventos convulsivos. Sin embargo, en pacientes con adecuado manejo de estatus epiléptico que persistan con deterioro de la función renal y el aumento de la CQ más allá del quinto día de seguimiento, indica evaluar diagnósticos diferenciales de RBM, ya que el pico de aumento de la CQ se ha descrito ocurre hacia el tercer día de finalizado el evento desencadenante de RBM, así como su retorno a valores normales hacia el quinto día, manteniéndose o incluso acelerándose este comportamiento farmacocinético en pacientes con requerimiento de terapia de reemplazo renal $(8,17-19)$. Dentro de los diagnósticos diferenciales de RBM, debe considerarse la presencia de efecto adverso a medicamento, que, para el caso aquí descrito, fue el LEV el único medicamento recibido por el paciente con evidencia científica de RBM como potencial efecto adverso. El tratamiento de la RBM por LEV no está estandarizado, sin embargo, debe basarse en la suspensión del medicamento, así como en el manejo de soporte con seguimiento estrecho clínico y paraclínico, esto, con base en la evidencia científica indirecta descrita para el tratamiento de la lesión renal aguda por RBM (17). Para el caso aquí descrito, fue tratamiento mencionado fue implementado exitosamente, observándose rápida mejoría de los niveles de CQ, recuperación progresiva de la función renal y egreso hospitalario con niveles normales de azoados y de CQ, y diagnóstico definitivo de RBM por LEV.

Se desconoce si existen factores de riesgo o desencadenantes para desarrollar RBM por LEV. El presente caso plantea la discusión de si el LEV debe ser utilizado frente a otros antiepilépticos en el contex to agudo, especialmente en el paciente crítico, ya que, al ser la RBM un efecto adverso que si bien tiene baja incidencia, de presentarse podría acarrear una importante carga en costos, estancia hospitalaria e incluso aumento de morbimortalidad. La falta de evidencia de los efectos adversos mayores del LEV, hace pertinente que la decisión de administrarlo continúe basándose en sus estudios de eficacia y seguridad (20-22), sin embargo, teniéndose en cuenta que dichos estudios son insuficientes para recomendar el uso de LEV como medicamento de elección en estatus epiléptico (23).

Los casos reportados de RBM por LEV dentro de los cuales la mayoría son en adultos y uno en niños, conllevo a que la sociedad europea incluyera dentro de los rótulos del medicamento este efecto adverso (24).

Con lo aquí descrito, se sugiere evaluar la suspensión del LEV en pacientes con lesión renal aguda y elevación de CQ persistentes a pesar del manejo adecuado de las potenciales causas de RBM.

En conclusión, la RBM por LEV es un efecto adverso a medicamento poco frecuente, que debe sospecharse en 
pacientes con lesión renal aguda y elevación de CQ persistentes a pesar de manejo adecuado de las potenciales causas de RBM.

\section{Conflictos de interés}

Los autores declaran no tener ningún conflicto de interés

\section{Financiación}

Recursos propios de los autores

\section{Referencias}

1. Lynch BA, Lambeng N, Nocka K, Kensel-Hammes P, Bajjalieh SM, Matagne A, et al. The synaptic vesicle protein SV2A is the binding site for the antiepileptic drug levetiracetam. Proc Natl Acad Sci [Internet]. 2004 Jun 29 [cited 2017 Oct 28];101 (26): 9861-6. Available from: http://www.ncbi.nlm.nih.gov/ pubmed/15210974

2. Crowder KM, Gunther JM, Jones TA, Hale BD, Zhang HZ, Peterson MR, et al. Abnormal neurotransmission in mice lacking synaptic vesicle protein $2 \mathrm{~A}$ (SV2A). Proc Natl Acad Sci U S A [Internet]. 1999 Dec 21 [cited 2017 Oct 28];96 (26):15268-73. Available from: http://www.ncbi.nlm.nih.gov/pubmed/10611374

3. Doelken MT, Hammen T, Bogner W, Mennecke A, Stadlbauer A, Boettcher U, et al. Alterations of intracerebral $\gamma$-aminobutyric acid (GABA) levels by titration with levetiracetam in patients with focal epilepsies. Epilepsia [Internet]. $2010 \mathrm{Mar}$ 19 [cited 2017 Oct 28]; 51 (8): 1477-82. Available from: http://www.ncbi.nlm. nih.gov/pubmed/20345933

4. Meehan AL, Yang X, Yuan L-L, Rothman SM. Levetiracetam has an activitydependent effect on inhibitory transmission. Epilepsia [Internet]. 2012 Mar [cited 2017 Oct 28]; 53 (3): 469-76. Available from: http://www.ncbi.nlm.nih.gov/ pubmed/22292611

5. Delanty N, Jones J, Tonner F. Adjunctive levetiracetam in children, adolescents, and adults with primary generalized seizures: Open-label, noncomparative, multicenter, long-term follow-up study. Epilepsia [Internet]. 2012 Jan [cited 2017 Oct 28];53 (1): 111-9. Available from: http://www.ncbi.nlm.nih.gov/ pubmed/22050371

6. Nicolson A, Lewis SA, Smith DF. A prospective analysis of the outcome of levetiracetam in clinical practice. Neurology [Internet]. 2004 Aug 10 [cited 2017 Oct 28]; 63 (3): 568-70. Available from: http://www.ncbi.nlm.nih.gov/pubmed/15304598

7. Akiyama H, Haga Y, Sasaki N, Yanagisawa T, Hasegawa Y. A case of rhabdomyolysis in which levetiracetam was suspected as the cause. Epilepsy Behav Case Reports [Internet]. 2014 [cited 2017 Oct 24]; (2): 152-5. Available from: http://www.ncbi.nlm.nih.gov/pubmed/25667895

8. Isaacson JE, Choe DJ, Doherty MJ. Creatine phosphokinase elevation exacerbated by levetiracetam therapy. Epilepsy Behav Case Reports [Internet]. 2014 [cited 2017 Oct 24]; (2): 189-91. Available from: http://www.ncbi.nlm.nih.gov/ pubmed/25667904

9. American Academy of Neurology. R, Li Y. Neurology. [Internet]. Vol. 88, Neurology. Advanstar Communications; 2017 [cited 2017 Oct 24]. P6.226. Available from: http://www.neurology.org/content/88/16_Supplement/P6.226

10. Di Lorenzo R, Li Y. Rhabdomyolysis associated with levetiracetam administration. Muscle Nerve [Internet]. 2017 Jul 1 [cited 2017 Oct 24];56 (1): E1-2. Available from: http://doi.wiley.com/10.1002/mus. 25548
11. Singh R, Patel DR, Pejka S. Rhabdomyolysis in a Hospitalized 16-Year-Old Boy: A Rarely Reported Underlying Cause. Case Rep Pediatr [Internet]. 2016 Nov 8 [cited 2017 Oct 24]; (2016): 1-2. Available from: https://www.hindawi. com/journals/cripe/2016/7873813/

12. Incecik F, Herguner OM, Besen S, Altunbasak S. Acute rhabdomyolysis associated with levetiracetam therapy in a child. Acta Neurol Belg [Internet]. 2016 Sep 23 [cited 2017 Oct 24]; 116 (3): 369-70. Available from: http://www.ncbi. nlm.nih.gov/pubmed/26399431

13. Omar MA, Wilson JP. FDA Adverse Event Reports on Statin-Associated Rhabdomyolysis. Ann Pharmacother [Internet]. 2002 Feb 26 [cited 2017 Oct 26]; 36 (2): 288-95. Available from: http://www.ncbi.nlm.nih.gov/pubmed/11847951

14. Thompson PD. Statin-Associated Myopathy. JAMA [Internet]. 2003 Apr 2 [cited 2017 Oct 26]; 289 (13): 1681. Available from: http://jama.jamanetwork.com/ article.aspx?doi=10.1001/jama.289.13.1681

15. Dalakas MC. Toxic and drug-induced myopathies. J Neurol Neurosurg Psychi [Internet]. 2009 Aug 1 [cited 2017 Oct 26];80 (8): 832-8. Available from: http:// www.ncbi.nlm.nih.gov/pubmed/19608783

16. Toxicidad antibacterianos: farmacocinética-farmacodinamia: prevención y manejo. Rev Méd Clín Las Condes [Internet]. 2014 May 1 [cited 2017 Oct 26]; 25 (3): 445-56. Available from: http://www.sciencedirect.com/science/article/pii/ S0716864014700616

17. Petejova N, Martinek A. Acute kidney injury due to rhabdomyolysis and renal replacement therapy: a critical review. Crit Care [Internet]. 2014 May 28 [cited 2017 Oct 26]; 18 (3): 224. Available from: http://www.ncbi.nlm.nih.gov/ pubmed/25043142

18. Maggi G, Quinteros Hinojosa F, Villagran MJ, Guasch Arévalo E, Gilsanz Rodríguez F. Renal Replacement Therapy in Acute Kidney Failure due to Rhabdomyolysis. Case Rep Crit Care [Internet]. 2012 Apr 26 [cited 2017 Oct 26]; (2012): 1-3. Available from: http://www.hindawi.com/journals/cricc/2012/603849/

19. Rubio A, Sanchez MI. Miopatías inducidas por fármacos. Papel del farmacéutico offarm. 2008; 27 (3): 80-6.

20. Chu-Shore C, Cormier CJ. Safety and efficacy of levetiracetam for the treatment of partial onset seizures in children from one month of age. Neuropsychiatr Dis Treat [Internet]. 2013 Feb [cited 2017 Oct 24]; (9): 295. Available from: http:// www.ncbi.nlm.nih.gov/pubmed/23458993

21. Abend NS, Monk HM, Licht DJ, Dlugos DJ. Intravenous levetiracetam in critically ill children with status epilepticus or acute repetitive seizures. Pediatr Crit Care Med [Internet]. 2009 Jul [cited 2017 Oct 24]; 10 (4): 505-10. Available from: http://content.wkhealth.com/linkback/openurl?sid=WKPTLP:landingpage \&an=00130478-200907000-00013

22. Aiguabella M, Falip M, Villanueva V, de la Peña P, Molins A, Garcia-Morales I, et al. Efficacy of intravenous levetiracetam as an add-on treatment in status epilepticus: A multicentric observational study. Seizure [Internet]. 2011 Jan [cited 2017 Oct 24]; 20 (1): 60-4. Available from: http://www.ncbi.nlm.nih.gov/ pubmed/21145758

23. Glauser T, Shinnar S, Gloss D, Alldredge B, Arya R, Bainbridge J, et al. Evidence-Based Guideline: Treatment of Convulsive Status Epilepticus in Children and Adults: Report of the Guideline Committee of the American Epilepsy Society. Epilepsy Curr [Internet]. 2016 [cited 2017 Oct 24]; 16 (1): 48-61. Available from: http://www.ncbi.nlm.nih.gov/pubmed/26900382

24. Anexo I Conclusiones científicas y motivos para la modificación de las condiciones de las autorizaciones de comercialización. [cited 2017 Oct 24]; Available from: https://ec.europa.eu/health/documents/community-register/2016/20161114136278/anx_136278_es.pdf 